\title{
RANS Modelling for Temperature Variance in Conjugate Heat Transfer
}

\author{
Gaoqiang Yang ${ }^{1,2}$, Hector Iacovides ${ }^{1}$, Timothy Craft $^{1}$, David Apsley ${ }^{1}$ \\ ${ }^{1}$ School of Mechanical, Aerospace and Civil Engineering \\ The University of Manchester \\ gaoqiang.yang@manchester.ac.uk; h.iacovides@manchester.ac.uk, tim.craft@manchester.ac.uk \\ ${ }^{2}$ FDII of China Aerodynamic Research and Development Center \\ MianYang, SiChuan, 621000, China
}

\begin{abstract}
We predict how turbulence generated temperature fluctuations propagate from the fluid regions into the adjacent walls. Within the RANS approach this is achieved by extending the transport equations for temperature variance and its dissipation rate across the solid walls which bound the flow region. The results of recent DNS studies of conjugate heat transfer under fully developed conditions in straight channels have been used to develop and optimize the model. Simulations of 1D fully developed channel flow (friction Reynolds number 150, Prandtl numbers 0.71 and 7) with heated solid wall are carried out and compared with DNS data, using an existing four-equation model proposed by Craft et al.[4] as a starting point. The transport equations for the two thermal parameters, temperature variance and its dissipation rate, are optimized by focusing on the decay of these two parameters in the solid region, using boundary values at the interface between the solid and fluid regions provided by DNS. Then a physically consistent and numerically stable set of interface conditions are developed to account for the jump in the value of temperature variance dissipation rate across the solid-fluid interface, caused by the different material thermal properties. Subsequent conjugate heat transfer simulations show that the proposed model is able to predict the correct distribution of the temperature variance within both the fluid and solid regions, for the entire range of thermal diffusivity and conductivity ratios between the fluid and solid regions and also for both fluid Prandtl numbers.
\end{abstract}

Keywords: Conjugate heat transfer, Turbulence models, Turbulent heat transfer, Temperature fluctuations.

\section{Introduction}

Conjugate heat transfer, the coupling of heat conduction in a solid and convective heat transfer in the adjacent fluid, is attracting increasing interest in many engineering applications. In particular, when flow is turbulent, the situation becomes rather complex. In such situations, there exist intense temperature fluctuations in the fluid due to the effects of the turbulent eddies. Temperature fluctuations in the near-wall turbulent fluid region can penetrate into the solid, leading to similar temperature fluctuations in the region adjacent to the fluid. These, in turn, can cause thermal stress fluctuations in the material, which may lead to thermal fatigue and then shorten the material's life (these research topics can be found[1-2], relating to thermal fatigue in $\mathrm{T}$-junctions). Thus it is necessary to develop an appropriate model to capture temperature fluctuations both in the near-wall fluid and the solid regions, which can provide guidance for engineering design.

Researches on temperature fluctuations for conjugate heat transfer based on RANS models are rather limited. One exception is the PhD study for fully developed channel flow with heated wall in Manchester[3], which has also been reported by Craft et al.[4] in 2010. In their research, an existing four-equation model (i.e. transport equations for the turbulent kinetic energy $k$, its dissipation rate $\varepsilon$, the temperature variance $\overline{\theta^{2}}$ and its dissipation rate $\varepsilon_{\theta}$ ) proposed by Hanjalić et al.[5] was extended to the solid region and comparisons with DNS data available at the time[6], led to the introduction of some modifications to the dissipation rate $(\varepsilon)$ equation and also to the temperature variance $\left(\overline{\theta^{2}}\right)$ equation and that of its dissipation rate $\left(\varepsilon_{\theta}\right)$. These modifications led to promising results for channel flow conjugate heat transfer. However, their study was confined to the case with a fixed ratio of thermal diffusivity $R_{\alpha}=\alpha_{f} / \alpha_{s}=1$ between fluid and solid, and various ratios of thermal conductivity, $R_{\lambda}=\lambda_{f} / \lambda_{s}$ under the condition of $\operatorname{Pr}=7$ (which corresponds to water at room temperature). More recently, DNS simulations for channel flow conjugate heat transfer with various values of $R_{\alpha}$ and $R_{\lambda}$ at $\operatorname{Re}=150$ and $\operatorname{Pr}=0.71$ have been reported in [7], which provide the distributions of the temperature variance and 
its dissipation rate across the fluid and the confining walls. Thus, it opens the possibility of testing the model developed by Craft et al.[4] over a wider range of conditions and make any necessary modifications.

\section{Cases studied}

The computational domain is one-dimensional turbulent channel flow with heated solid wall, as shown in Figure 1. The friction Reynolds number $\left(\operatorname{Re}_{\tau}\right)$ based on half channel width $(H)$ is 150 and the Prandtl number ( $\operatorname{Pr}$ ) is either 7, or 0.71. The dimensionless wall thickness $\left(d^{+}=u_{\tau} d / v\right.$, where $u_{\tau}$ is the friction velocity) is 20 for $\operatorname{Pr}=7$ and 150 for $\operatorname{Pr}=0.71$. Both conjugate heat transfer and non-conjugate, decoupled, heat transfer cases are studied. For conjugate cases, different combinations of thermal diffusivity ratios $\left(R_{\alpha}=0.5,1,2\right)$ and thermal conductivity ratios $\left(R_{\lambda}=0.5,1,2\right)$ are tested.

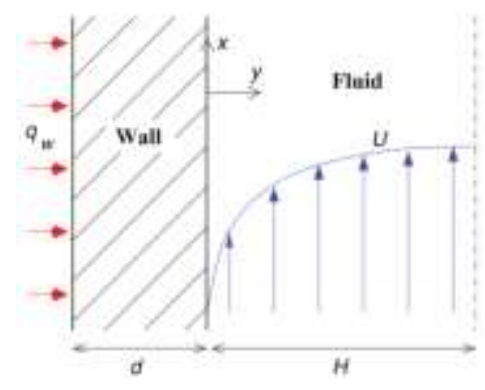

Fig. 1: Geometry of fully developed turbulent channel flow with heated wall.

\section{Turbulence modelling}

The starting point of this study is the model from Craft et al.[4], which resulted in a promising prediction for fully developed channel flow with heated wall at $\operatorname{Pr}=7$.

\subsection{Model descriptions}

The RANS equations needed to be solved for incompressible flow are as follows:

$$
\begin{gathered}
\frac{\partial U_{i}}{\partial x_{i}}=0, \quad \frac{D\left(U_{i}\right)}{D t}=\frac{\partial}{\partial x_{j}}\left[v \frac{\partial U_{i}}{\partial x_{j}}-\overline{u_{i} u_{j}}\right]-\frac{1}{\rho} \frac{\partial P}{\partial x_{i}} \\
\frac{D \Theta}{D t}=\frac{\partial}{\partial x_{j}}\left[\alpha \frac{\partial \Theta}{\partial x_{j}}-\overline{u_{j} \theta}\right]
\end{gathered}
$$

where $U_{i}, P$ and $\Theta$ are the mean velocity, pressure and temperature respectively, and $\alpha$ is the thermal diffusivity, and $u_{i}$ and $\theta$ are the velocity and temperature fluctuations. The Reynolds stress can be obtained based on eddy-viscosity approximation, while turbulent heat fluxes can be obtained using a simple eddy-diffusivity formulation:

$$
\overline{u_{i} u_{j}}=\frac{2}{3} k \delta_{i j}-v_{t}\left(\frac{\partial U_{i}}{\partial x_{j}}+\frac{\partial U_{j}}{\partial x_{i}}\right), \quad \overline{u_{i} \theta}=-\frac{v_{t}}{\sigma_{\theta}} \frac{\partial \Theta}{\partial x_{i}}
$$

where $\sigma_{\theta}=0.9, v_{t}=c_{\mu} f_{\mu} k^{2} / \widetilde{\varepsilon}$, with $\widetilde{\varepsilon}=\varepsilon-2 v\left(\partial k^{0.5} / \partial x_{j}\right)^{2} . c_{\mu}$ and $f_{\mu}$ are expressed as:

$$
c_{\mu}=\min \left[0.09, \frac{1.2}{1+3.5 \eta+f_{R S}}\right], f_{\mu}=1-\exp \left[-\left(\operatorname{Re}_{t} / 90\right)^{1 / 2}-\left(\operatorname{Re}_{t} / 400\right)^{2}\right], \eta=\max (\tilde{S}, \tilde{\Omega}), \operatorname{Re}_{t}=\frac{k^{2}}{v \tilde{\varepsilon}}
$$




$$
\begin{gathered}
f_{R S}=0.235[\max (0, \eta-3.333)]^{2}\left[\exp \left(-\mathrm{Re}_{t} / 400\right)+\sqrt{S_{I}^{2}}\right], \tilde{S}=\frac{k}{\tilde{\varepsilon}} \sqrt{\frac{1}{2} S_{i j} S_{i j}}, \tilde{\Omega}=\frac{k}{\widetilde{\varepsilon}} \sqrt{\frac{1}{2} \Omega_{i j} \Omega_{i j}} \\
S_{I}=\left(S_{i j} S_{j k} S_{k i}\right) /\left(S_{n l} S_{n l} / 2\right)^{3 / 2}, S_{i j}=\frac{\partial U_{i}}{\partial x_{j}}+\frac{\partial U_{j}}{\partial x_{i}}, \Omega_{i j}=\frac{\partial U_{i}}{\partial x_{j}}-\frac{\partial U_{j}}{\partial x_{i}}
\end{gathered}
$$

The four-equation model $\left(k-\varepsilon-\overline{\theta^{2}}-\varepsilon_{\theta}\right)$ from Craft et al.[4] is written as follows:

$$
\begin{gathered}
\frac{D k}{D t}=P_{k}-\varepsilon+\frac{\partial}{\partial x_{j}}\left[\left(v+f_{g} v_{t}\right) \frac{\partial k}{\partial x_{j}}\right] \\
\frac{D \widetilde{\varepsilon}}{D t}=c_{\varepsilon 1} \frac{\tilde{\varepsilon} P_{k}}{k}-c_{\varepsilon 2} f_{\varepsilon} \frac{\widetilde{\varepsilon}^{2}}{k}+\frac{\partial}{\partial x_{j}}\left[\left(v+f_{g} \frac{v_{t}}{\sigma_{\varepsilon}}\right) \frac{\partial \widetilde{\varepsilon}}{\partial x_{j}}\right]+S_{\varepsilon}+E_{\varepsilon} \\
\frac{D \overline{\theta^{2}}}{D t}=2 P_{\theta}-2 \varepsilon_{\theta}+\frac{\partial}{\partial x_{j}}\left[\left(\frac{v}{\operatorname{Pr}}+f_{g} \frac{v_{t}}{\sigma_{\theta}}\right)\right] \frac{\partial \overline{\theta^{2}}}{\partial x_{j}} \\
\frac{D \widetilde{\varepsilon}_{\theta}}{D t}=c_{\varepsilon_{\theta 1}} \frac{P_{k} \widetilde{\varepsilon}_{\theta}}{k}+c_{\varepsilon_{\theta 3}} \frac{P_{\theta} \widetilde{\varepsilon}_{\theta}}{\theta^{2}}-c_{\varepsilon_{\theta 4}} \frac{\widetilde{\varepsilon}_{\theta}^{2}}{\overline{\theta^{2}}}-c_{\varepsilon_{\theta 2}} \frac{\tilde{\varepsilon} \widetilde{\varepsilon}_{\theta}}{k}+\frac{\partial}{\partial x_{j}}\left[\left(\frac{v}{\operatorname{Pr}}+f_{g} \frac{v_{t}}{\sigma_{\theta} \sigma_{\varepsilon_{\theta}}}\right) \frac{\partial \widetilde{\varepsilon}_{\theta}}{\partial x_{j}}\right]+S_{\varepsilon_{\theta}}+E_{\theta}+S_{d}
\end{gathered}
$$

For dynamic field models, $P_{k}=-\overline{u_{i} u_{j}} \partial U_{i} / \partial x_{j}$ is the generation rate. $S_{\varepsilon}$ and $E_{\varepsilon}$ are low-Re source terms only influential in the near-wall fluid region. For thermal field models, the generation rate is $P_{\theta}=-\overline{u_{i}} \theta \partial T / \partial x_{i}$ while as in the dynamic models, $S_{\varepsilon_{\theta}}, E_{\theta}$ and $S_{d}$ are the source terms used to balance the molecular diffusion in the near wall fluid region as follows:

$$
S_{\varepsilon_{\theta}}=-0.8\left(\varepsilon_{\theta}-\widetilde{\varepsilon}_{\theta}\right) \frac{\tilde{\varepsilon}}{k} \exp \left(-\frac{\operatorname{Re}_{t}^{2}}{c_{n 1}}\right), E_{\theta}=c_{\varepsilon \theta} \alpha \alpha_{t}\left(\frac{\partial^{2} T}{\partial x_{j} \partial x_{k}}\right)^{2}, S_{d}=-\frac{\partial}{\partial x_{j}}\left[c_{d} \alpha\left[1-\left(\frac{\varepsilon_{\theta}-\tilde{\varepsilon}_{\theta}}{\varepsilon_{\theta}}\right)\right] \frac{\partial \tilde{\varepsilon}_{\theta}}{\partial x_{j}}\right]
$$

where the coefficients $c_{n}, \quad c_{\varepsilon \Theta}$ and $c_{d}$ are 10, 1.5 and 0.7 respectively. Dissipation rate $\varepsilon_{\theta}$ is expressed as $\varepsilon_{\theta}=\widetilde{\varepsilon}_{\theta}+\alpha\left(\partial \bar{\theta}^{2}{ }^{0.5} / \partial x_{j}\right)^{2}$, in which $\alpha$ is the thermal diffusivity.

When extending thermal model equations (9) and (10) to the solid wall, terms related to the dynamic field, such as production terms and other terms containing dynamic time scales have no meaning and thus are left out, while the fluid thermal properties are replaced with the solid ones. As a consequence, the thermal model equations take the following form:

$$
\frac{D \overline{\theta^{2}}}{D t}=-2 \varepsilon_{\theta}+\frac{\partial}{\partial x_{j}}\left[\alpha \frac{\partial \overline{\theta^{2}}}{\partial x_{j}}\right], \quad \frac{D \widetilde{\varepsilon}_{\theta}}{D t}=-c_{\varepsilon \theta 4} \frac{\widetilde{\varepsilon}_{\theta}^{2}}{\overline{\theta^{2}}}+\frac{\partial}{\partial x_{j}}\left[\alpha \frac{\partial \widetilde{\varepsilon}_{\theta}}{\partial x_{j}}\right]+S_{d}
$$


In practical problems, thermal properties between fluid and solid are usually not the same, leading to non-continuous dissipation rate $\varepsilon_{\theta}$ across the fluid-solid interface. To account for this discontinuity, the model assumes that the variable $\tilde{\varepsilon}_{\theta}$ is continuous across the interface. As a result, the jump of dissipation rate $\varepsilon_{\theta}$ across the interface can be written:

$$
\varepsilon_{\theta, w f}-\varepsilon_{\theta, w s}=\alpha_{f}\left(\frac{\partial \sqrt{\overline{\theta^{2}}}}{\partial x_{j}}\right)_{w f}^{2}-\alpha_{s}\left(\frac{\partial \sqrt{\overline{\theta^{2}}}}{\partial x_{j}}\right)_{w s}^{2}
$$

where subscript $w f$ is short for wall fluid side, $w s$ wall solid side. The coefficients in this model are listed in table 1.

Table 1: Model coefficients.

\begin{tabular}{|c|c|c|c|c|c|c|c|c|c|c|c|}
\hline$c_{\varepsilon 1}$ & $c_{\varepsilon 2}$ & $\sigma_{\varepsilon}$ & $c_{\varepsilon \theta 1}$ & $c_{\varepsilon \theta 2}$ & $c_{\varepsilon \theta}$ & $c_{\varepsilon \theta 3}$ & $c_{\varepsilon \theta 4}$ & $\sigma_{\theta}$ & $\sigma_{\varepsilon \theta}$ & $c_{E 1}$ & $c_{E 2}$ \\
\hline 1.44 & 1.92 & 1.3 & 0.72 & 0.8 & 1.5 & 1.3 & 2.2 & 0.9 & 1.3 & 0.0022 & -0.7 \\
\hline
\end{tabular}

\subsection{Numerical method}

The computational domain has been discretized based on finite volume method and a 1D numerical solver has been developed using Fortran. In the fluid region, the number of grids is 140 with an expansion ratio 1.025, while in the solid region 100 grids has been used, which has been shown in the previous study to ensure grid-independence of the results. At the centre of channel flow, symmetric boundary condition has been used. At the outer edge of the wall, a fixed amount of heat flux has been prescribed. The mean temperature and temperature variance are continuous across the fluid-solid interface, and also the mean heat flux and the heat flux fluctuation.

\section{Model development}

The DNS data that became available since the study of [4] have not only highlighted the need for further model development, but have also provided additional validation data that can be used to address the predictive weaknesses. In particular, the availability of data for the temperature variance and its dissipation rate across the solid region provide the opportunity to first develop the thermal fluctuation transport model for the solid region and then couple it to the fluid side for further development. Consequently, in this Section, thermal transport equations in the solid region are first considered separately from those in the fluid region. Then the modelling of the near wall turbulence is addressed by coupling the fluid and solid regions together. The final development, involves the development of a new interface condition.

\subsection{Development within the solid region}

The transport equations for the thermal fluctuations in the solid region has been shown in 3.1. (here $S_{d}$ is removed, because the destruction rate of $\widetilde{\varepsilon}_{\theta}$ will be re-optimized). Noting that in these equations only the value of one constant $c_{\varepsilon \theta 4}$ needs be determined, we prescribe the surface values of $\overline{\theta^{2}}$ and $\varepsilon_{\theta}$ from the DNS data and then carry simulations across the solid region. After several numerical experiments over a range of cases with different solid thermal properties $\left(\mathrm{R}_{\alpha}=0.5\right.$, 1,$\left.2 ; \mathrm{R}_{\lambda}=0.5,1,2\right)$, the optimum value of $c_{\varepsilon \theta 4}$ was finally determined as 8.47 while the original value in [4] was 2.2. The resulting comparisons are shown in Figure 2. 

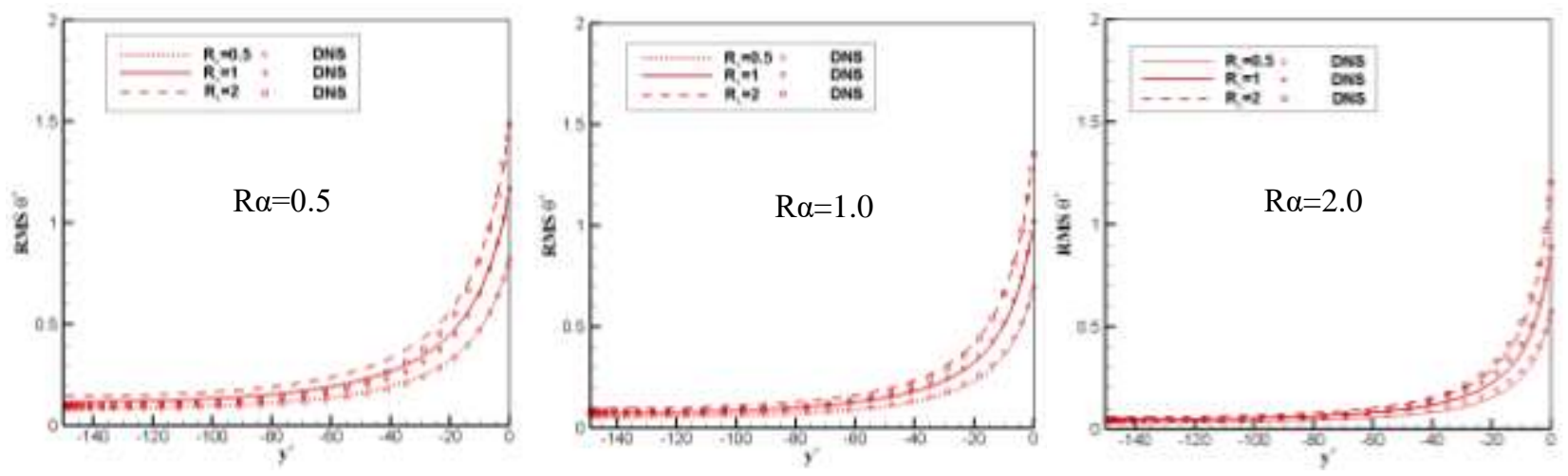

Fig. 2: Temperature fluctuations obtained by only solving thermal transport equations in the solid.

\subsection{Development within fluid region}

After the development and validation of the transport equations for the temperature variance and its dissipation rate within the solid region, the resulting model has been used to simulate the conjugate heat transfer cases.

The results for cases at $\operatorname{Re}_{\tau}=150$ and for $\operatorname{Pr}=7$ and 0.71 are shown in Figure 3. As seen from these figures, over a range of thermal property ratios the temperature variance around the interface is significantly over-estimated. Since, as shown in Section 4.1, the model performs well in the solid region when the correct interface values of the temperature variance and its dissipation rate are prescribed, this weakness is attributed to the predictions on the fluid side. In addition, since the predictions for both isothermal and isoflux cases from the original model in [4] have been already in good agreement with relevant DNS data, the modification should be active only in the fluid region for conjugate cases.
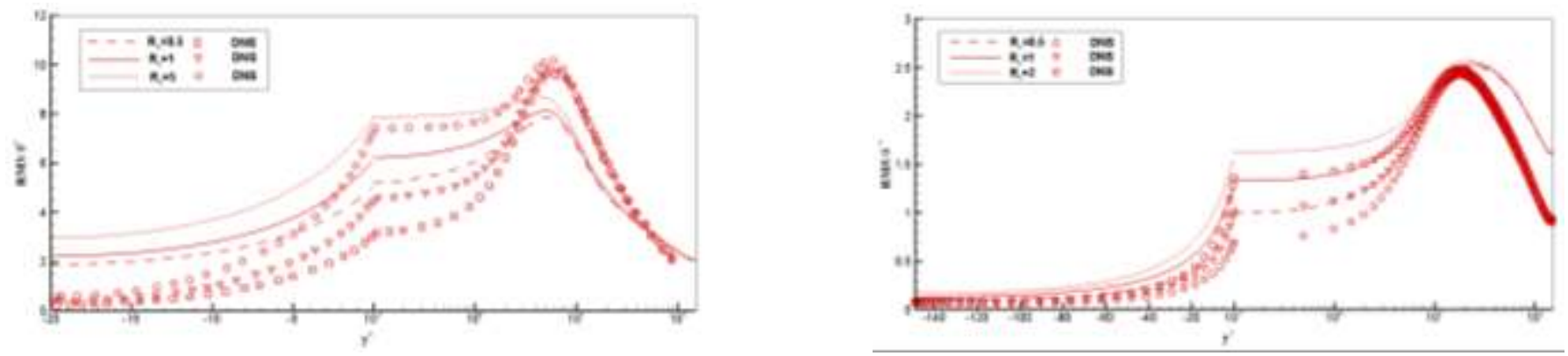

Fig. 3: Temperature fluctuations under $\operatorname{Re}_{\tau}=150$ with $R_{\alpha}=1$ (left: $\operatorname{Pr}=7$, right: $\operatorname{Pr}=0.71$ ).

The way to decrease the temperature variance level at the near-wall fluid region is to increase its dissipation rate. In the near-wall fluid region, the two source terms $\left(P_{k}\right.$ and $\left.P_{\theta}\right)$ in the equation (11) diminish near the wall. Examining then the two remaining sink terms, the first one includes the thermal time scale and the second one the dynamic time scale. For all conjugate cases, the dynamic time scale is prominent, which means that the only option, is to reduce the second sink term near the wall and thus increase the dissipation rate of the temperature variance. Based on this analysis and numerical experiments, a new expression for $c_{\varepsilon_{\theta 2}}$ is proposed as follows:

$$
c_{\varepsilon \theta 2}=0.8-\underbrace{12.53 \operatorname{Pr}^{0.126}}_{\text {term } 0} \underbrace{\exp \left(-\operatorname{Re}_{t} / 15.0\right)}_{\text {term } 1} \underbrace{\left[\exp \left(-\frac{\tilde{\varepsilon}_{\theta}}{\varepsilon_{\theta}}\right)-0.367\right]\left[1.0-\exp \left(-\frac{\tilde{\varepsilon}_{\theta}}{\varepsilon_{\theta}}\right)\right]}_{\text {term } 2}
$$

Since original value of $c_{\varepsilon \theta 2}$ was determined by considering the decay rate of temperature fluctuations in the decaying grid turbulence, term 1 in the equation (15) can ensure that the modified coefficient value recovers to this original one, i.e. 0.8, away from the wall and the proposed modification is only significant at the near-wall region. The modification will be only 
significantly active for conjugate heat transfer due to term 2 , since the value of term 2 is zero for limiting cases in the nearwall fluid region, because the ratio $\widetilde{\varepsilon}_{\theta} / \varepsilon_{\theta}$ equals to one for the isoflux case and zero for the isothermal case. Another feature of this modification is that, term0 incorporates the effects of Pr number, since the thickness of thermal conduction sub-layer varies with the Pr number. The values of the constant in term0 is determined through numerical experiments by considering both $\operatorname{Pr}=0.71$ and $\operatorname{Pr}=7$.

Figure 4 shows comparisons which result from the introduction of the form of $c_{\varepsilon \theta 2}$ described by equation (15) for the non-conjugate cases. It can be seen that the modifications have little effect on these cases. The results from both the previous model and also current modified model are in close accord with DNS data.

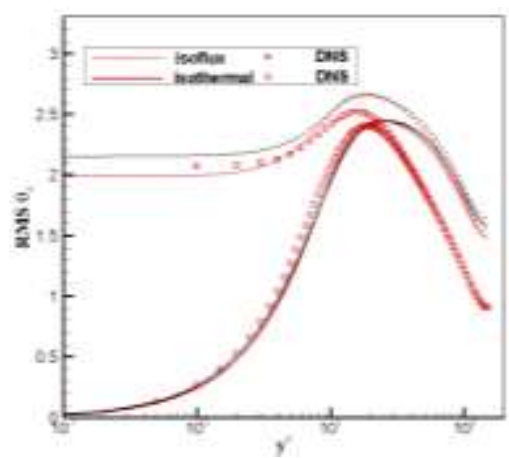

Fig. 4: Temperature variance for two limiting cases at $\operatorname{Re}_{\tau}=150, \operatorname{Pr}=0.71$.(Red: current results, black: original model [4]).

Predictions for temperature variance in conjugate heat transfer problems at $\operatorname{Pr}=0.71$ with various thermal diffusivity and conductivity ratios can be seen in Figures 5-6. They show that the modified model has significantly improved the predictions in the solid region for all conjugate cases, while keeping the predictions in the near-wall fluid region still close to the DNS data. For cases with $\mathrm{R}_{\alpha}=2$, however, the decay rate is still somewhat stronger than that of the DNS data, while for cases with $\mathrm{R}_{\alpha}=0.5$ and $\mathrm{R}_{\lambda}=2$ the RANS-predicted decay rate is rather weaker.
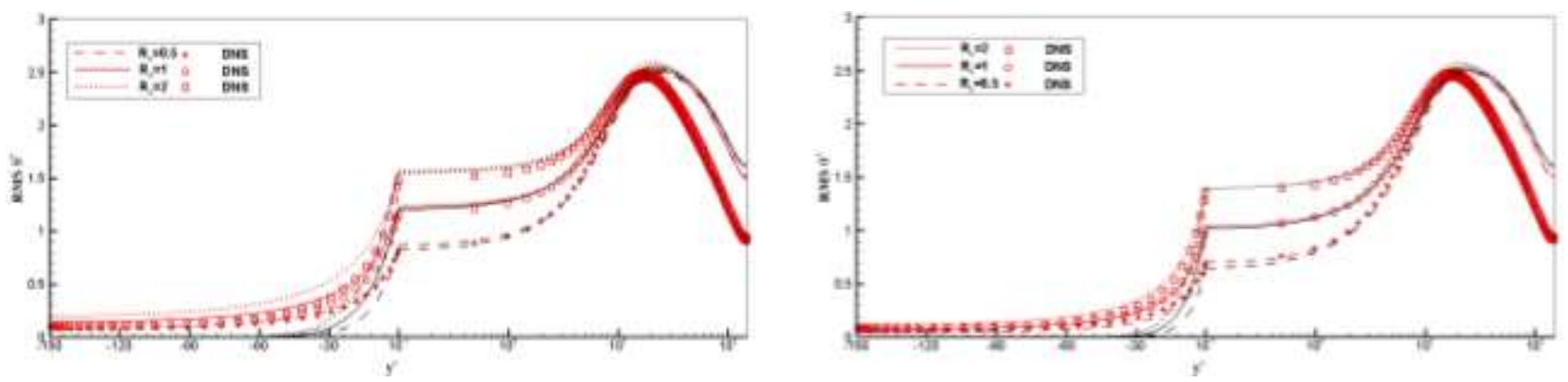

Fig. 5: Predictions for temperature variance across the fluid and solid regions at $\operatorname{Re}_{\tau}=150, \operatorname{Pr}=0.71$ (red: current results, black: original model[4]; left: $\mathrm{R}_{\alpha}=0.5$, right: $\mathrm{R}_{\alpha}=1$ ). 


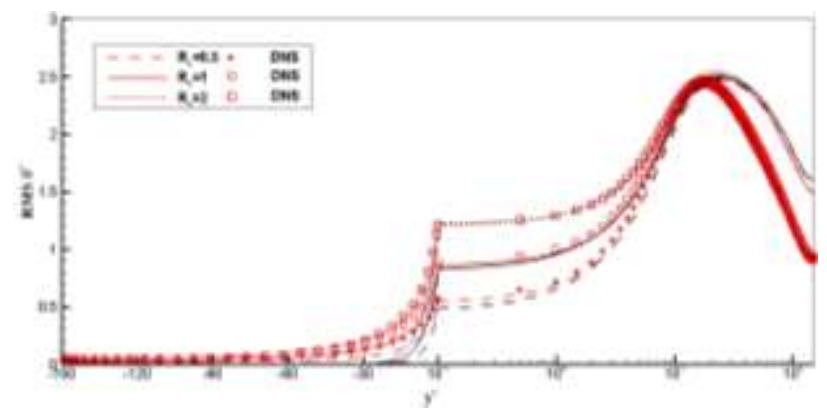

Fig. 6: Predictions for temperature variance across the fluid and solid regions at $\operatorname{Re}_{\tau}=150, \operatorname{Pr}=0.71$ with $\mathrm{R}_{\alpha}=2$ (red: current results, black: original model[4]).

Predictions for $\operatorname{Pr}=7$ are shown in Figure 7 compared with the corresponding DNS data. It can be seen that the predictions for the temperature variance in the near-wall fluid region are improved for nearly all cases. In the solid region, predictions for cases with lower values of $R_{\lambda}$ are improved significantly. For cases with $R_{\lambda}=5$ and 100 , however, the decay rates are slower than those in the DNS data. This weakness was traced to the interface condition for the dissipation rate of the temperature variance $\varepsilon_{\theta}$ across the interface, a feature of this model which is re-visited in the next section.
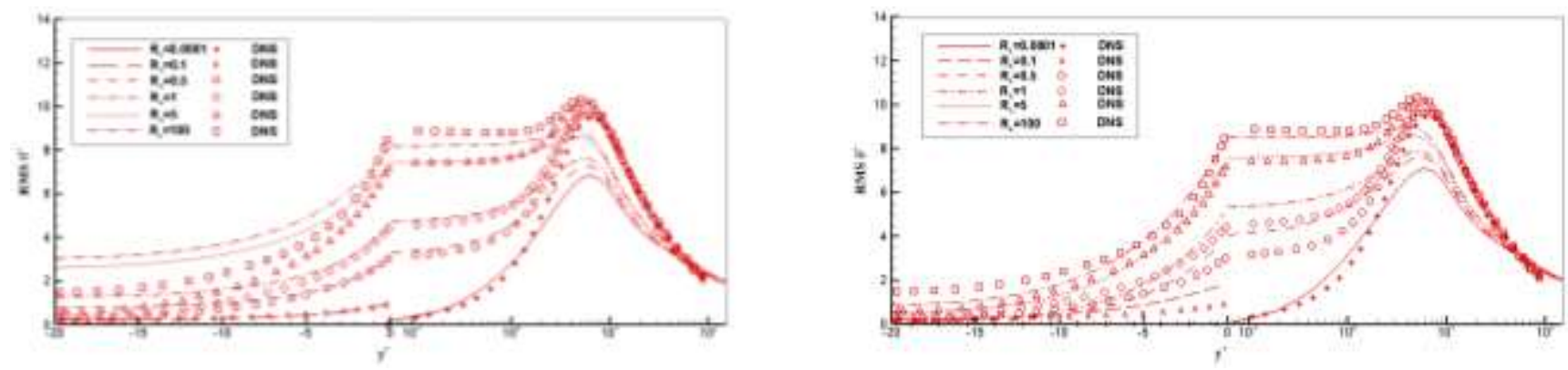

Fig. 7: Predictions for temperature variance across the fluid and solid regions at $\operatorname{Re}_{\tau}=150, \operatorname{Pr}=7$ with $\operatorname{R} \alpha=1$ (left: current results, right: original model[4]).

\subsection{Development for interface condition}

As pointed out in Section 3.1, the interface condition from the model [4] is as follows:

$$
\varepsilon_{\theta, w f}-\varepsilon_{\theta, w s}=\alpha_{f}\left(\frac{\partial \sqrt{\overline{\theta^{2}}}}{\partial x_{j}}\right)_{w f}^{2}-\alpha_{s}\left(\frac{\partial \sqrt{\overline{\theta^{2}}}}{\partial x_{j}}\right)_{w s}^{2}
$$

Figure 8 (left) presents the difference between the jump predicted using the above interface model and the jump present in the DNS data. It shows that for all cases the jump from the model is much lower than that of DNS data, which means that the changing rate of temperature variance in the near-wall fluid region could not penetrate correctly into the solid. It, thus, is necessary to devise an appropriate interface model to give the correct jump, in order to improve further the numerical predictions. Moreover, when conjugate heat transfer systems approach the isothermal state, equation (16) can recover to the most often used boundary condition, namely that for the non-conjugate isothermal problems, as follows: 


$$
\varepsilon_{\theta, w f}=\alpha_{f}\left(\frac{\partial \sqrt{\overline{\theta^{2}}}}{\partial x_{j}}\right)_{w f}^{2}
$$

which has been proved reasonable by both wall-limiting behaviour analysis and DNS data[8]. As a consequence, the subsequent modification takes this point into account. As a result, a new interface model is proposed as follows:

$$
\varepsilon_{\theta, w f}-\varepsilon_{\theta, w s}=f \bullet\left[\alpha_{f}\left(\frac{\partial \sqrt{\overline{\theta^{2}}}}{\partial x_{j}}\right)_{w f}^{2}-\alpha_{s}\left(\frac{\partial \sqrt{\overline{\theta^{2}}}}{\partial x_{j}}\right)_{w s}^{2}\right]
$$

where $f$ is introduced to correct the jump according to DNS data and $f$ is dependent on thermal properties of fluid and solid:

$$
f=\min \left[4.5 \tanh \left(4 \frac{R_{\lambda}^{2}}{R_{\alpha}}\right)+1, \quad 2.5\right]
$$

Predictions for the jump from this new interface model are shown in Figure 8(right). The new interface model provides more accurate jump of the temperature variance dissipation rate across the fluid-solid interface than the previous model.
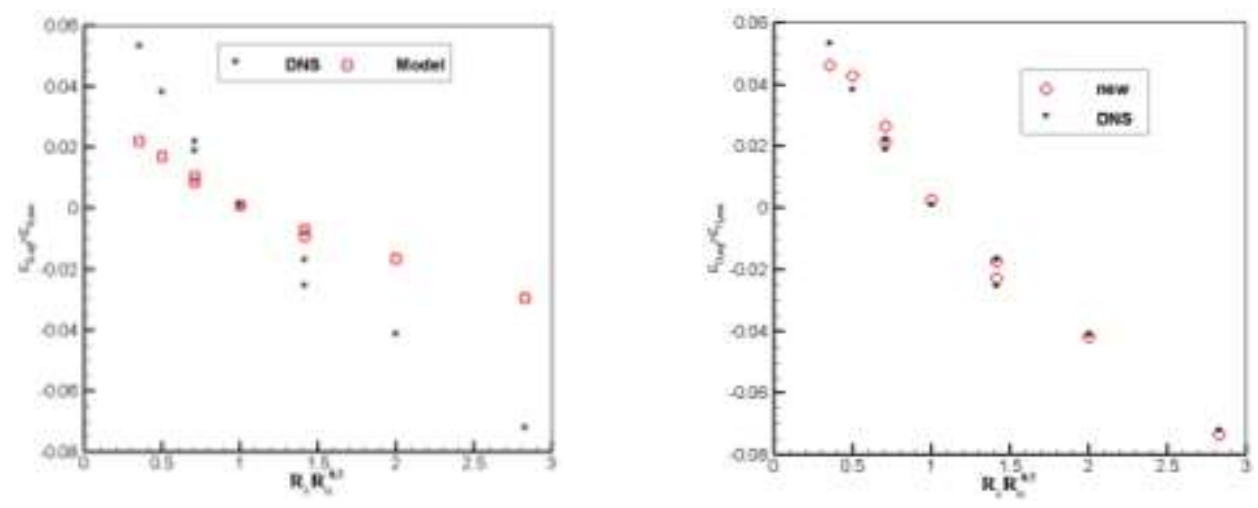

Fig. 8: Predictions for the jump of dissipation rate $\varepsilon_{\theta}$ compared with DNS data(left: original model[4], right: current model).

The final results for temperature variance in conjugate heat transfer are shown in Figure 9 for $\operatorname{Pr}=0.71$ and Figure 10 for $\operatorname{Pr}=7$ after incorporating the new interface model. It can be seen, from Figure 9, that the final modified model can predict temperature variance quite well compared with DNS data for conjugate heat transfer problems with various fluid and solid thermal properties at $\operatorname{Pr}=0.71$. As for $\operatorname{Pr}=7$, the results are still improved by the current modified model compared with that from the previous model, as seen from Figure 10. In particular, the predictions for temperature variance across the interface and in the near-wall fluid region are improved significantly now for $\operatorname{Pr}=7$. 


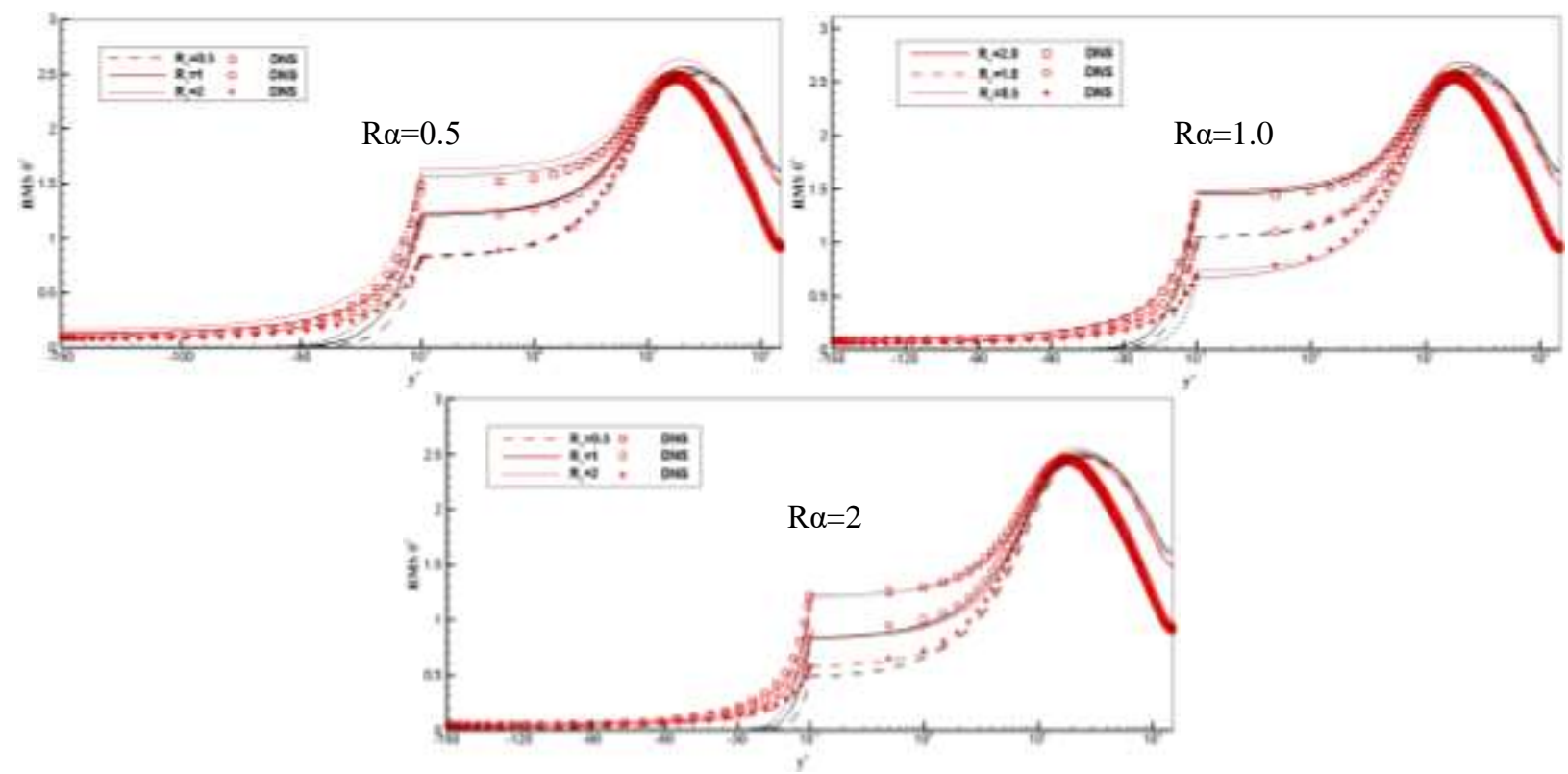

Fig. 9: Predictions for temperature variance at $\operatorname{Re}_{\tau}=150, \operatorname{Pr}=0.71$ (red: current results, black: original model, symbols: DNS).
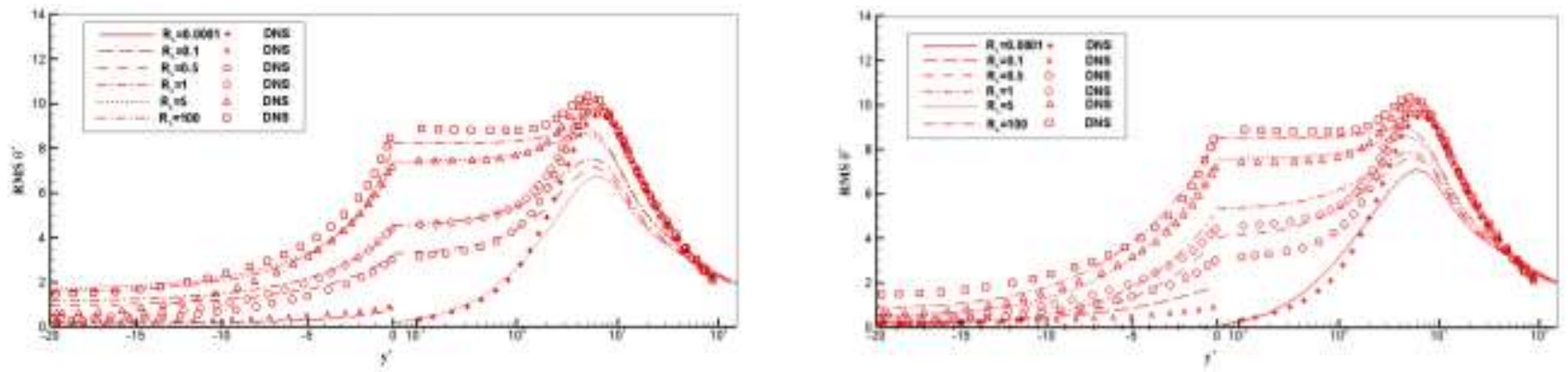

Fig. 10: Predictions for temperature variance at $\operatorname{Re}_{\tau}=150, \operatorname{Pr}=7$ at fixed $\mathrm{R}_{\alpha}=1$.(right: current results, left: original model[4]).

\section{Conclusion}

This study has focused on the prediction of the temperature variance in the conjugate heat transfer problems using RANS modelling as a frame work. A series of model modifications in the fluid and solid regions are made by reference to recently available DNS data. In addition, a new interface model which can accurately represent the jump of temperature variance dissipation rate across the solid-fluid interface has been proposed. The final modified model has shown that the predictions for the temperature variance have been improved significantly for both $\operatorname{Pr}=0.71$ and $\operatorname{Pr}=7$.

The next stage of the study is to test this proposed model against more challenging and complex 3D problems. One of them is the channel flow with a round, heated jet at its bottom, which has been reported in [9] using DNS.

\section{References}

[1] J. Westin, F. Alavyoon, P. Andersson, P. Veber, M. Henriksson, and C. Andersson, "Experiments and unsteady CFD calculations of thermal mixing in a T-junction," in OECD/NEA/IAEA Workshop on Benchmarking of CFD Codes for Application to Nuclear Reactor Safety (CFD4NRS), Munich, pp. 1-15, 2006.

[2] Y. Odemark, T. Green, K. Angele, J. Westin, F. Alavyoon, and S. Lundström, "High-cycle thermal fatigue in mixing tees: New large eddy simulations validated against new data obtained by PIV in the Vattenfall experiment," in 17th International Conference on Nuclear Engineering, Brussels, pp. 775-785, Paper ICONE17-175962, 2009. 
[3] S. Uapipatanakul, "Development of computational methods for conjugate heat transfer analysis in complex industrial applications," Ph.D. dissertation, School of MACE, The University of Manchester.

[4] T. J. Craft, H. Iacovides, S. Uapipatanakul, "Towards the development of RANS models for conjugate heat transfer," Journal of Turbulence, vol. 11, no. 26, pp. 1-16, 2010.

[5] K. Hanjalić, S. Kenjeres, F. Durst, "Natural convection in partitioned two-dimensional enclosures at higher Reyleigh numbers, International Journal of Heat and Mass Transfer," Int. J. Heat Mass Transfer, vol. 39, no. 7, pp. 1407-1427, 1996.

[6] I. Tiselj, R. Bergant, B. Mavko, I. Bajsić et al., "DNS of turbulent heat transfer in channel flow with heat conduction in the solid wall," Journal of Heat Transfer, Vol. 123: 849-857, $2001 \mathrm{~b}$.

[7] C. Flageul, S. Benhamadouche, É. Lamballais, D. Laurence, "On the discontinuity of the dissipation rate associated with the temperature variance at the fluid-solid interface for cases with conjugate heat transfer," International Journal of Heat and Mass Transfer, vol. 111: 321-328, 2017.

[8] B.E. Launder and N. D. Sandham, "Closure strategies for turbulent and transitional flows," Cambridge University Press 2002.

[9] Z. Wu, D. Laurence, H. Iacovides, I. Afgan, "Direct simulation of conjugate heat transfer of jet in channel crossflow," International Journal of Heat and Mass Transfer, Vol. 110: 193-208, 2017. 